\title{
MHC Haplotype-dependent Regulation of MOG-induced EAE in Rats
}

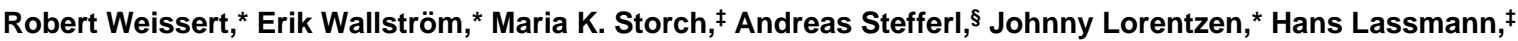 \\ Christopher Linington, $\$$ and Tomas Olsson* \\ *Neuroimmunology Unit, Center of Molecular Medicine L8:04, Karolinska Hospital, S-17176 Stockholm, Sweden; ${ }^{\ddagger}$ Neurological Institute, \\ University of Vienna, A-1090 Vienna, Austria; and ${ }^{\S}$ Max-Planck-Institute for Neurobiology, D-82152 Martinsried, Germany
}

\begin{abstract}
Experimental autoimmune encephalomyelitis (EAE) induced in the rat by active immunization with myelin-oligodendrocyte-glycoprotein (MOG) is mediated by synergy between MOG-specific $T$ cells and demyelinating MOGspecific antibody responses. The resulting disease is chronic and displays demyelinating central nervous system (CNS) pathology that closely resembles multiple sclerosis. We analyzed major histocompatibility complex (MHC) haplotype influences on this disease. The MHC haplotype does not exert an all-or-none effect on disease susceptibility. Rather, it determines the degree of disease susceptibility, recruitment of MOG-specific immunocompetent cells, clinical course, and CNS pathology in a hierarchical and allele-specific manner. Major haplotype-specific effects on MOG-EAE map to the MHC class II gene region, but this effect is modified by other MHC genes. In addition, non-MHC genes directly influence both disease and $\mathrm{T}$ cell functions, such as the secretion of IFN- $\gamma$. Thus, in MOG-EAE, allelic MHC class II effects are graded, strongly modified by other MHC genes, and overcome by effects of non-MHC genes and environment. (J. Clin. Invest. 1998. 102:1265-1273.) Key words: MHC • $\mathrm{EAE} \bullet$ myelin-oligodendrocyte-glycoprotein $\bullet$ interferon- $\gamma \bullet$ rat
\end{abstract}

\section{Introduction}

Human organ-specific inflammatory diseases such as multiple sclerosis (MS) ${ }^{1}$ result from environmental influences interacting with multiple genes (1). The major histocompatibility complex (MHC) is well established as one of the gene regions predisposing to MS (2). However, the particular genes within the MHC that are involved and the mechanisms by which they act are still controversial (3). The MHC influence on the suscepti-

Address correspondence to Dr. Robert Weissert, Department of Neurology, University of Tübingen, 72076 Tübingen, Germany. Phone: +49-7071-2982141; FAX: +49-7071-295260; E-mail: robert. weissert@uni-tuebingen.de

Received for publication 6 February 1998 and accepted in revised form 20 July 1998.

1. Abbreviations used in this paper: $\mathrm{CM}$, complete medium; $\mathrm{CNS}$, central nervous system; ConA, concanavalin A; EAE, experimental autoimmune encephalomyelitis; LN, lymph nodes; MBP, myelin basic protein; MHC, major histocompatibility complex; MNC, mononuclear cells; MOG, myelin-oligodendrocyte glycoprotein; MS, multiple sclerosis; p.i., post immunization; RT, room temperature; RT1, MHC of rat; SI, stimulation index.

J. Clin. Invest.

(C) The American Society for Clinical Investigation, Inc. 0021-9738/98/09/1265/09 \$2.00

Volume 102, Number 6, September 1998, 1265-1273

http://www.jci.org bility to experimental autoimmune encephalomyelitis (EAE) induced by myelin basic protein (MBP), which is a purely $\mathrm{T}$ cell-dependent disease, has been studied extensively (4). In this model, certain MHC haplotypes are commonly described as disease permissive, while others are described as resistant. This can be considered a gross oversimplification of the situation in human organ-specific inflammatory diseases such as MS and type I diabetes, in which the autoimmune response involves both $\mathrm{T}$ cells and a variety of autoantibody responses (5-7). Such a more diverse autoimmune response may well initiate and/or include a large number of different immune effector mechanisms, which, in turn, are regulated by genes encoded both within and outside the MHC. This might modify the relative importance of specific MHC class II molecules in determining disease susceptibility.

We use myelin-oligodendrocyte-glycoprotein (MOG)induced EAE in rats to study how differences in the MHC haplotypes influence a model that incorporates more of the complexity in immune effector mechanisms seen in human organ-specific inflammatory diseases. In MOG-EAE, a specific $\mathrm{T}$ cell response triggers an inflammatory cascade that opens the blood-brain barrier to circulating anti-MOG specific antibodies and serum complement. Antibody can then bind to the outer surface of the myelin sheath and mediate demyelination through a combination of complement and antibody-dependent cellular cytotoxicity-mediated mechanisms, while the local production of complement-derived pro-inflammatory factors amplifies the local inflammatory response. The histopathology of the lesions induced by this combination of immune effector mechanisms closely resembles that seen in MS $(8,9$, the present work). To study the MHC in MOG-EAE on a constant non-MHC background and disease-inducing environment, we used MHC-congenic and intra-MHC recombinant congenic rats. The disease-inducing environmental challenge is titrated on two different $\mathrm{MHC}$ haplotypes. To investigate nonMHC gene modifying effects on the MHC, we varied the nonMHC background while holding the MHC haplotype and the disease-inducing environment constant. Clinical course, histopathology, and MOG-specific immunity are studied in relation to these manipulations.

\section{Methods}

Animals. Female rats 10-14 weeks of age were used in all experiments. All strains have been described (10) (see Table I). ACI rats were originally obtained from Harlan Sprague Dawley Inc. (Indianapolis, IN); PVG-RT1 ${ }^{\mathrm{a}}$ and BN rats from Harlan UK Limited (Blackthorn, UK); DA, DA.1H, LEW, LEW.1A, LEW.1AV1, and LEW.1W from the Zentralinstitut für Versuchstierzucht (Hannover, Germany); and LEW.1N, LEW.1AR1, LEW.1AR2, LEW.1WR1, and LEW.1WR2 from Prof. H. Hedrich (Medizinische Hochschule, Hannover, Germany). Rats were then locally bred in filter boxes and routinely tested for specific pathogens. Breeding pairs were checked for homozygosity by examination of a microsatellite marker located within the RT1 region. The experiments were approved by the local ethical committee. 
Immunogens and Ags. The N-terminal sequences of rat MOG (amino acids 1-125) and rat $\mathrm{S} 100 \beta$ (11) were expressed in Escherichia coli and purified to homogeneity by chelate chromatography (12). The purified proteins in $6 \mathrm{M}$ urea were then dialyzed against PBS to obtain preparations that were stored at $-20^{\circ} \mathrm{C}$. MOG and $\mathrm{S} 100 \beta$ were used in all cell cultures at $3 \mu \mathrm{g} / \mathrm{ml}$. These Ag concentrations had given optimal stimulations in previous titration experiments. Concanavalin A (ConA) (Sigma Chemical Co., St. Louis, MO) was used at $1 \mu \mathrm{g} / \mathrm{ml}$.

Induction and evaluation of EAE. Rats were anesthetized and injected intradermally at the base of the tail with $100 \mu \mathrm{l}$ of inoculum containing $50 \mu \mathrm{g}$ of rat MOG in saline emulsified (1:1) with CFA (Sigma Chemical Co., St. Louis, MO) containing $200 \mu \mathrm{g}$ of Mycobacterium tuberculosis (strain H 37 RA; Difco Laboratories, Detroit, MI). For dose titration experiments in LEW.1A and LEW.1N rats, individual rats were injected with $1 \mu \mathrm{g}, 5 \mu \mathrm{g}, 10 \mu \mathrm{g}, 20 \mu \mathrm{g}, 50 \mu \mathrm{g}$, or $100 \mu \mathrm{g}$ of MOG mixed with CFA as described above.

Rats were scored for clinical signs of EAE and were weighed daily up to $40 \mathrm{~d}$ post immunization (p.i.) by two alternating investigators. The signs were scored as follows: Grade 1, tail weakness or tail paralysis; and Grade 2, hind leg paraparesis or hemiparesis; Grade 3, hind leg paralysis or hemiparalysis; Grade 4, complete paralysis (tetraplegy), moribund state, or death. Ataxia was routinely assessed. A disease remission was defined as an improvement in disease score from either 3 or 4 to 1 , or from 2, 3, or 4 to 0 that was maintained for at least $2 \mathrm{~d}$ consecutively. A relapse was defined as an increase in the clinical deficit of at least two points that lasted for at least two days.

Histopathological examination. Histological evaluation was performed on paraformaldehyde-fixed, paraffin-embedded sections of brains and spinal cords sampled at day 12 p.i. and day 40 p.i. Paraffin sections were stained with hematoxylin-eosin, Luxol fast blue, and Bielschowsky silver impregnation to assess inflammation, demyelination, and axonal pathology, respectively.

The inflammatory index was determined from the number of perivascular inflammatory infiltrates of each animal on an average of 15 complete cross sections of spinal cord. The degree of demyelination was evaluated separately for brain and spinal cord sections and semiquantitatively described and scored as follows: traces of perivascular or subpial demyelination (0.5); marked perivascular or subpial demyelination (1); confluent perivascular or subpial demyelination (2); massive confluent demyelination (e.g., half of spinal cord, one optic nerve complete) (3); and extensive demyelination (transverse myelitis, half of the cerebellar white matter or more, both optic nerves complete) (4).

In adjacent serial sections, immunohistochemistry was performed with $\mathrm{Ab}$ against the following targets: macrophages/activated microglia (ED1; Serotec, Oxford, UK), T cells (W3/13; Seralab, Sussex, UK), C9 (13), rat Ig (biotinylated $\alpha$-rat; Amersham, Buckinghamshire, UK), and glial fibrillary acidic protein (GFAP; Boehringer Mannheim, Mannheim, Germany). Bound primary Ab was detected with a biotin-avidin technique previously described in detail (14). Control sections were incubated in the absence of primary Ab or with non-immune rabbit serum.

Fractionation and cultivation of mononuclear cells from $L N$ and blood. Under deep anesthesia, draining inguinal (lymph nodes [LN]) were dissected out and put into DMEM (Life Technologies, Paisley, Scotland). LN were disrupted and mononuclear cells (MNC) washed twice in DMEM, resuspended in complete medium (CM) containing DMEM supplemented with $1 \%$ rat serum, $1 \%$ penicillin/streptomycin (Life Technologies), 1\% glutamine (Life Technologies), and 50 $\mu \mathrm{M}$ 2-ME (Life Technologies), and flushed through a 70- $\mu$ m plastic strainer (Falcon; Becton Dickinson-Vacutainer Systems, Franklin Lakes, CA). MNC were cultured at a concentration of $2 \times 10^{6}$ cells/ $\mathrm{ml}$ in either 96-well round-bottom microtiter plates (Nunc, Roskilde, Denmark) with $100 \mu \mathrm{l}$ of cell suspension per well, or 24-well flat-bottom plates (Falcon; Becton Dickinson-Vacutainer Systems) with $1000 \mu \mathrm{l}$ of cell suspension per well at $37^{\circ} \mathrm{C}$ in a humidified atmosphere containing $5 \% \mathrm{CO}_{2}$.
Assays of Ag-induced proliferation and IFN- $\gamma$ production. Proliferative experiments were performed in triplicates. $2 \times 10^{5} \mathrm{MNC} /$ well in $100 \mu \mathrm{l}$ of $\mathrm{CM}$ were cultured in 96-well round-bottom microtiter plates with or without $\mathrm{Ag}$ for $60 \mathrm{~h}$ and pulsed with $0.5 \mathrm{mCi}\left[{ }^{3} \mathrm{H}\right] \mathrm{TdR}$ (Amersham, Stockholm, Sweden) per well for $12 \mathrm{~h}$. DNA was collected on glass fiber filters (Skatron Instruments Inc., Sterling, VA) and $\left[{ }^{3} \mathrm{H}\right] \mathrm{TdR}$-incorporation was measured in a beta counter (Beckman Instruments Inc., Palo Alto, CA). To assess any $\mathrm{T}$ cell proliferation induced by minor bacterial contamination of the recombinant MOG, recombinant $\mathrm{S} 100 \beta$ was added to parallel $\mathrm{T}$ cell cultures. The MOGspecific proliferative stimulation index (SI) was obtained with the formula $1+\left(\mathrm{SI}_{\mathrm{MOG}}-\mathrm{SI}_{\mathrm{S} 100 \beta}\right)$.

Ag-induced IFN- $\gamma$ mRNA expression was measured by culture of $2 \times 10^{6} \mathrm{MNC}$ in $1000 \mu \mathrm{l}$ of $\mathrm{CM}$ per well in 24-well plates in the presence and absence of MOG, S100 $\beta$, and ConA for $48 \mathrm{~h}$ at $37^{\circ} \mathrm{C}$ and $5 \%$ $\mathrm{CO}_{2}$. The cells were collected, washed twice in PBS (Life Technologies), and counted, and $1 \times 10^{5}$ cells were dried onto restricted areas of glass slides (Probe On; Fisher Scientific Co., Pittsburgh, PA) for each Ag. In situ hybridization and counting of positive cells were performed as described (15). Responses induced by bacterial contamination of the recombinant MOG were controlled by using recombinant S100 $\beta$ protein. The numbers of IFN- $\gamma$ mRNA-expressing cells induced by recombinant $S 100 \beta$ were $0-4$ per $1 \times 10^{5}$ cells. The MOGspecific numbers of IFN- $\gamma$ mRNA-expressing cells were expressed as MOG minus S100 $\beta$ IFN- $\gamma$ mRNA-expressing cells.

An Elispot method was used to enumerate T cells secreting IFN- $\gamma$ after Ag exposure (15). Nitrocellulose-bottom 96-well plates (MAHA; Millipore, Molsheim, France) were coated with the mAb DB1 (antirat IFN- $\gamma$; a generous gift of Dr. Peter van der Meide, TNO Primate Centre, Rijswijk, The Netherlands). After blocking with DMEM containing 5\% FCS (Life Technologies), we added triplicates of $4 \times 10^{5}$ cells in $200 \mu \mathrm{l}$ of $\mathrm{CM}$ per well and antigen to the plates and incubated them for $48 \mathrm{~h}$ at $37^{\circ} \mathrm{C}$ in a humidified atmosphere containing $5 \% \mathrm{CO}_{2}$. Secreted and bound IFN- $\gamma$ was visualized with biotinylated mDB12 (anti-rat IFN- $\gamma$; Dr. Peter van der Meide), avidin-biotin peroxidase (Vector Laboratories Inc., Burlingame, CA), and staining with carbazole (Sigma Chemical Co.). In control cultures, S100 $\beta$ induced 0-1 cell per $4 \times 10^{5}$ cells of IFN- $\gamma$-secreting cells. The MOG-specific numbers of IFN- $\gamma$-secreting cells were expressed as MOG minus S100 $\beta$ IFN- $\gamma$-secreting cells.

Enumeration of cells secreting Ag-specific antibodies. An Elispot method was used for enumeration of plasma cells secreting antiMOG specific antibodies (16). Nitrocellulose-bottom 96-well plates (MAHA; Millipore) were coated for $12 \mathrm{~h}$ with either $3 \mu \mathrm{g} / \mathrm{ml}$ MOG in PBS, $3 \mu \mathrm{g} / \mathrm{ml} \mathrm{S} 100 \beta$ in PBS, or PBS alone. The plates were then washed with PBS and blocked with DMEM containing 5\% FCS (Life Technologies). Triplicates of $2 \times 10^{5}$ MNC per well in $100 \mu \mathrm{l}$ of CM for each antigen were incubated for $16 \mathrm{~h}$ at $37^{\circ} \mathrm{C}$ in a humidified atmosphere containing $5 \% \mathrm{CO}_{2}$. Secreted and bound antibodies were visualized by polyclonal rabbit anti-rat Ig (Dako, Glostrup, Denmark), biotinylated anti-rabbit Ig (Dako) followed by avidin-biotin peroxidase (Vector Laboratories), and staining with carbazole (Sigma Chemical Co.). The number of $\mathrm{S} 100 \beta$-specific spots was equal to the number of spots in PBS-coated wells. The mean number of spots in PBS-coated control wells was subtracted from the number of spots recorded in the MOG-coated wells. The results are expressed as the number of anti-MOG Ab-secreting cells per $10^{5} \mathrm{MNC}$.

Collection of serum samples and ELISA. Blood samples for $\mathrm{Ab}$ measurements were taken at days 12 p.i. and 40 p.i. 96 -well ELISA plates (Nunc) were coated with $2.5 \mu \mathrm{g} / \mathrm{ml}(100 \mu \mathrm{l} /$ well) recombinant rat MOG overnight at $4^{\circ} \mathrm{C}$. Plates were washed with $\mathrm{PBS} / 0.05 \%$ Tween and blocked for $1 \mathrm{~h}$ at room temperature (RT). Diluted serum samples were added after washing and plates were incubated for $1 \mathrm{~h}$ at RT. Then, plates were washed, and diluted rabbit-anti-rat antiserum (Nordic, Tilburg, The Netherlands) was added and incubated for $1 \mathrm{~h}$ at RT. Unbound antibodies were then removed by washing prior to the addition of peroxidase-conjugated goat-anti-rabbit antiserum (Nordic) diluted in PBS/0.05\% Tween (1:10,000). After $30 \mathrm{~min}$, incu- 
bation plates were washed thoroughly, and bound antibodies were vi-

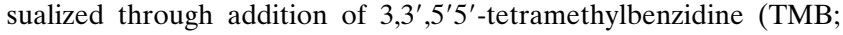
Sigma Chemical Co.). The enzymatic reaction was stopped with $1 \mathrm{M}$ $\mathrm{HCl}$ after $15 \mathrm{~min}$ of incubation in darkness, and the optical density was read at $450 \mathrm{~nm}$.

Statistics. Differences in the occurrence of inflammation and demyelination between MOG-immunized strains were tested for significance with Fischer's exact test. T and B cell responses were tested for significance by comparing responses in MOG-immunized rats with responses in CFA-immunized rats, using Student's $t$ test for variables with normal distribution and the Mann-Whitney U test for other variables. Normality testing was performed with the Shapiro-Wilk test. Corrections for multiple comparisons were made with the Bonferroni method.

\section{Results}

Modulation of MOG-EAE by RT1 haplotypes on a constant non-MHC LEW background. We examined how different RT1 haplotypes in combination with LEW non-MHC genes affected clinical and histopathological disease and the MOGspecific immune response. For this purpose, we used a series of MHC congenic LEW rat strains $\left(\mathrm{RT}^{\mathrm{n}}, \mathrm{RT}^{\mathrm{a}}{ }^{\mathrm{a}}, \mathrm{RT}^{\mathrm{av} 1}, \mathrm{RT}^{\mathrm{u}}\right.$, and $\mathrm{RT}^{\mathrm{l}}$ ) selected to provide the greatest possible variety of MHC class II alleles (17).

Disease outcome in these LEW RT1 congenic rat strains ranked from mild or no disease to acute lethal disease (Fig. 1 $A)$. The most fulminant disease course was seen in LEW.1N $\left(\mathrm{RT}^{\mathrm{n}}\right)$ rats, which developed early onset acute lethal disease with paraplegia, ataxia, and extensive demyelinating plaques. LEW.1A (RT1 $\left.{ }^{\mathrm{a}}\right)$ and LEW.1AV1 $\left(\mathrm{RT}^{\mathrm{av} 1}\right)$ rats showed a chronic and/or relapsing type of disease. Some LEW.1W $\left(\mathrm{RT}^{\mathrm{u}}\right)$ rats displayed clinical signs at a late time point, while LEW $\left(\mathrm{RT} 1^{1}\right)$ rats remained free from clinical signs.

The intensity of the histopathological lesions paralleled the severity of clinical signs (Fig. 2, see Fig. 5). The hyperacute fatal disease course in LEW.1N rats was associated with widespread inflammatory demyelinating lesions in the CNS at day 12 p.i. These actively demyelinating lesions contained large numbers of polymorphonuclear cells, as well as $\mathrm{T}$ cells and macrophages, and were associated with extensive deposition of IgG and C9. LEW.1AV1 and LEW.1A rats exhibited a mixture of purely inflammatory and mixed inflammatory-demyelinating lesions on day 12 p.i., which by day 40 p.i. had progressed to form large areas of confluent demyelination. Only one out of thirteen LEW.1W rats was found to have a low degree of inflammatory pathology in the CNS on day 12 p.i., although by day 40 p.i., several rats exhibited a subclinical inflammatory response in the CNS that was in some cases accompanied by minor perivascular demyelination. However, the two most severely diseased LEW.1W rats were found to have large confluent focal demyelinating lesions in the spinal cord, medulla oblongata, and cerebellum. In accordance with the lack of clinical disease in LEW rats, no CNS pathology was observed on day 12 p.i. and only a small number of rats exhibited moderate perivascular inflammation in the spinal cord on day 40 p.i.

We studied if the RT1 haplotype-regulated disease outcome was correlated to any qualitative or quantitative aspect of the MOG-specific immune response on day 12 p.i. (Fig. 3 $A$ ). MOG-induced T cell proliferative responses and the number of IFN- $\gamma$ mRNA-expressing cells in MNC from the draining LN correlated with the severity of clinical disease. When com-

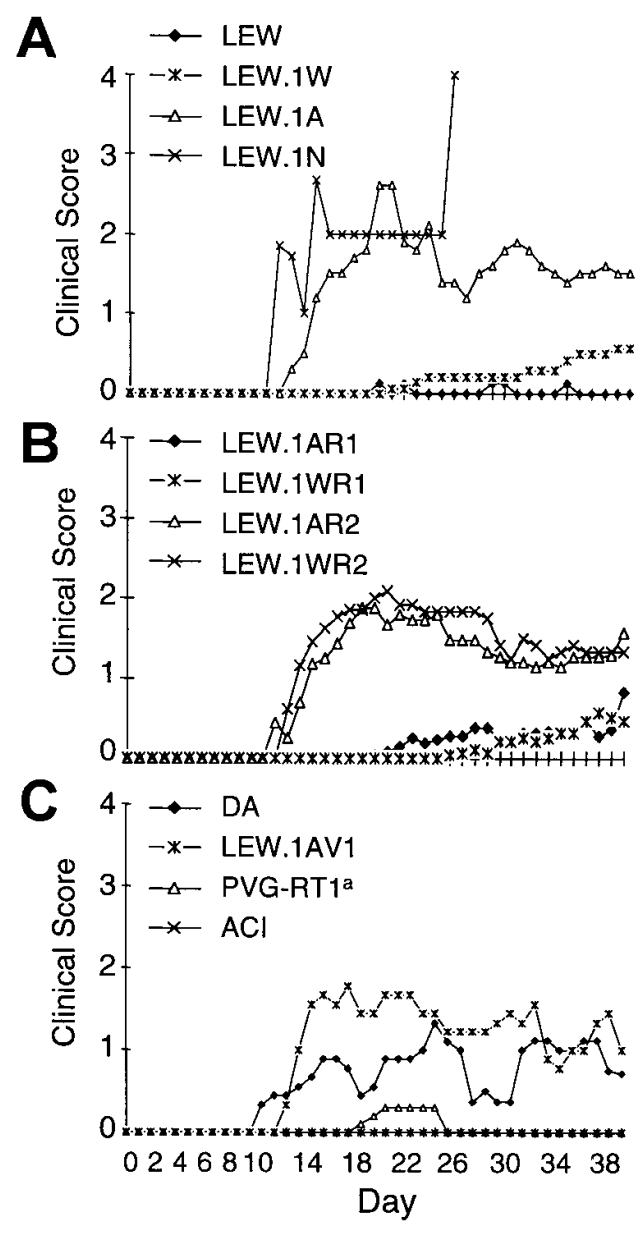

Figure 1. Clinical course of MOG-induced EAE in MHC congenic and intra-MHC recombinant congenic rat strains. Mean daily scores of $(A)$ LEW $(n=8)$, LEW.1W $(n=14)$, LEW.1A $(n=10)$, and LEW.1N $(n=14) ;(B)$ LEW.1AR1 $(n=27)$, LEW.1WR1 $(n=28)$, LEW.1AR2 $(n=18)$, and LEW.1WR2 $(n=13) ;(C)$ DA $(n=9)$, LEW.1AV1 $(n=10)$, PVG-RT1 ${ }^{\text {a }}(n=10)$, and ACI $(n=13)$ rats. LEW.1N rats developed an acute lethal disease with ataxia and paraplegia (13 of 14 rats died between days $12-15$ p.i.). LEW.1A, LEW.1AR2, and LEW.1WR2 rats had chronic disease, while DA and LEW.1AV1 rats had chronic and/or relapsing disease. LEW.1W, LEW.1AR1, LEW.1WR1, and PVG-RT1 ${ }^{\mathrm{a}}$ rats were semiprotected. LEW and ACI rats showed no clinical signs of disease during the $40 \mathrm{~d}$ observation period.

pared with CFA-injected controls, LEW.1N rats displayed the highest stimulation indices, followed by LEW.1A, LEW.1W, and LEW rats. A similar hierarchy was apparent for the number of MOG-induced IFN- $\gamma$ mRNA-expressing cells with the highest numbers in the LEW.1N strain, followed by LEW.1A, LEW.1W, and LEW rats. All strains showed similarly high proliferation indices and high numbers of IFN- $\gamma$ mRNAexpressing cells after mitogenic stimulation with ConA (data not shown). The degree of the MOG-specific B cell response was also similarly ranked in that the number of anti-MOG $\mathrm{Ab}$ secreting cells was high in LEW.1N and LEW.1A, while LEW.1W and LEW had low numbers. This was paralleled by the anti-MOG Ab titers. Thus, the onset of clinical disease and lesion growth/activity in LEW RT1 congenic rat strains corre- 
Day 12
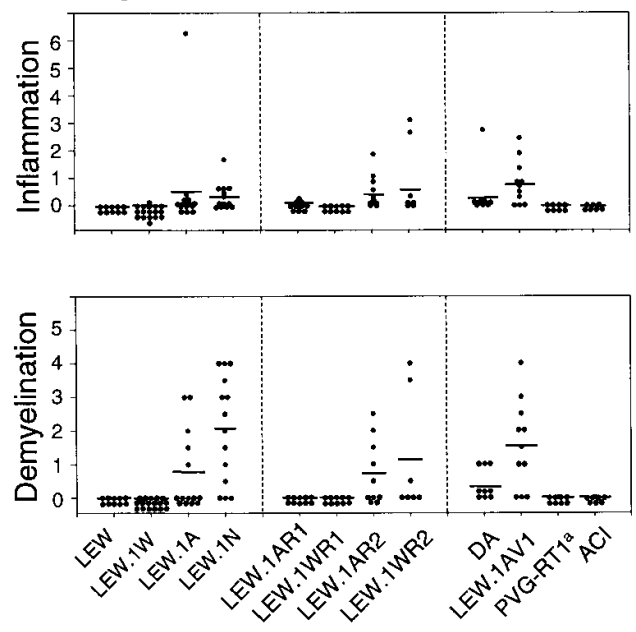

Day 40
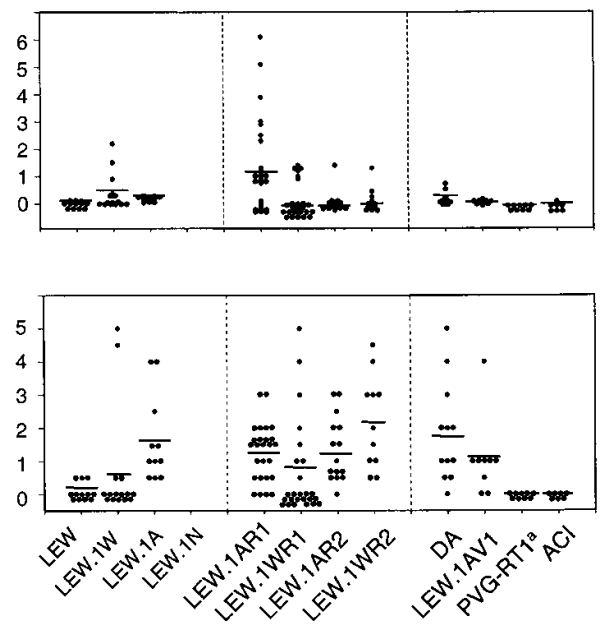

Figure 2. Semiquantitative assessment of CNS inflammation and demyelination. Individual rats sacrificed on day 12 p.i. or day 40 p.i. are represented by black dots and group means by horizontal lines. At day 12 p.i., inflammation was more frequent in LEW.1A $(P=0.002)$, LEW.1AR2 $(P<0.001)$, LEW.1WR2 $(P=$ $0.003)$, and LEW.1N $(P<0.001)$ rats compared with LEW.1W rats, and demyelination was more frequent in LEW.1A $(P=0.048)$, LEW.1AR2 $(P=0.012)$, and LEW.1N $(P<0.001)$ rats than in LEW. $1 \mathrm{~W}$ rats. At day 40 p.i., no differences remained in regard to inflammation in rats with the LEW background $(P>0.05)$, but demyelination was more frequent in LEW.1A $(P=0.001)$, LEW.1AR1 $(P=0.003)$, LEW.1AR2 $(P=0.03)$, and LEW.1WR2 $(P=0.001)$ rats compared with LEW.1W rats. When comparing rats sharing the RT1 ${ }^{\text {av1 }}$ haplotype but having different non-MHC background genomes, we found that inflammation and demyelination were less frequent in PVG-RT1 a and ACI rats at day 12 p.i. (inflammation, $P=0.010$ and 0.012 ; demyelination, $P=0.010$ and 0.012 ) and day 40 p.i. (inflammation, $P=0.001$ and 0.026 ; demyelination, $P=0.002$ and 0.007$)$, but not in DA rats $(P>0.05$ for all variables and timepoints), compared with LEW.1AV1 rats. All $P$ values were calculated with Fischer's exact test corrected for multiple comparisons with the Bonferroni method.

lated to the degree of functional activation and expansion of MOG-specific T and B cells.

$R T 1$ determines the Ag concentration threshold necessary to trigger disease. The effect of the RT1 haplotype on determining sensitivity to disease induction (eg. environment) was investigated in the highly susceptible MHC congenic strains LEW.1N and LEW.1A. Animals were immunized with doses of MOG ranging from $1 \mu \mathrm{g}$ to $100 \mu \mathrm{g}$ MOG. LEW.1N rats developed both clinical disease and CNS pathology at all $\mathrm{Ag}$ doses tested, whereas LEW.1A rats only developed disease when injected with $\geq 50 \mu \mathrm{g}$ of MOG (Fig. $4, A$ and $B$ ). Interestingly, not only disease severity but also disease course were dose related, with the majority of LEW.1N rats developing a chronic, rather than acute, disease when injected with lower

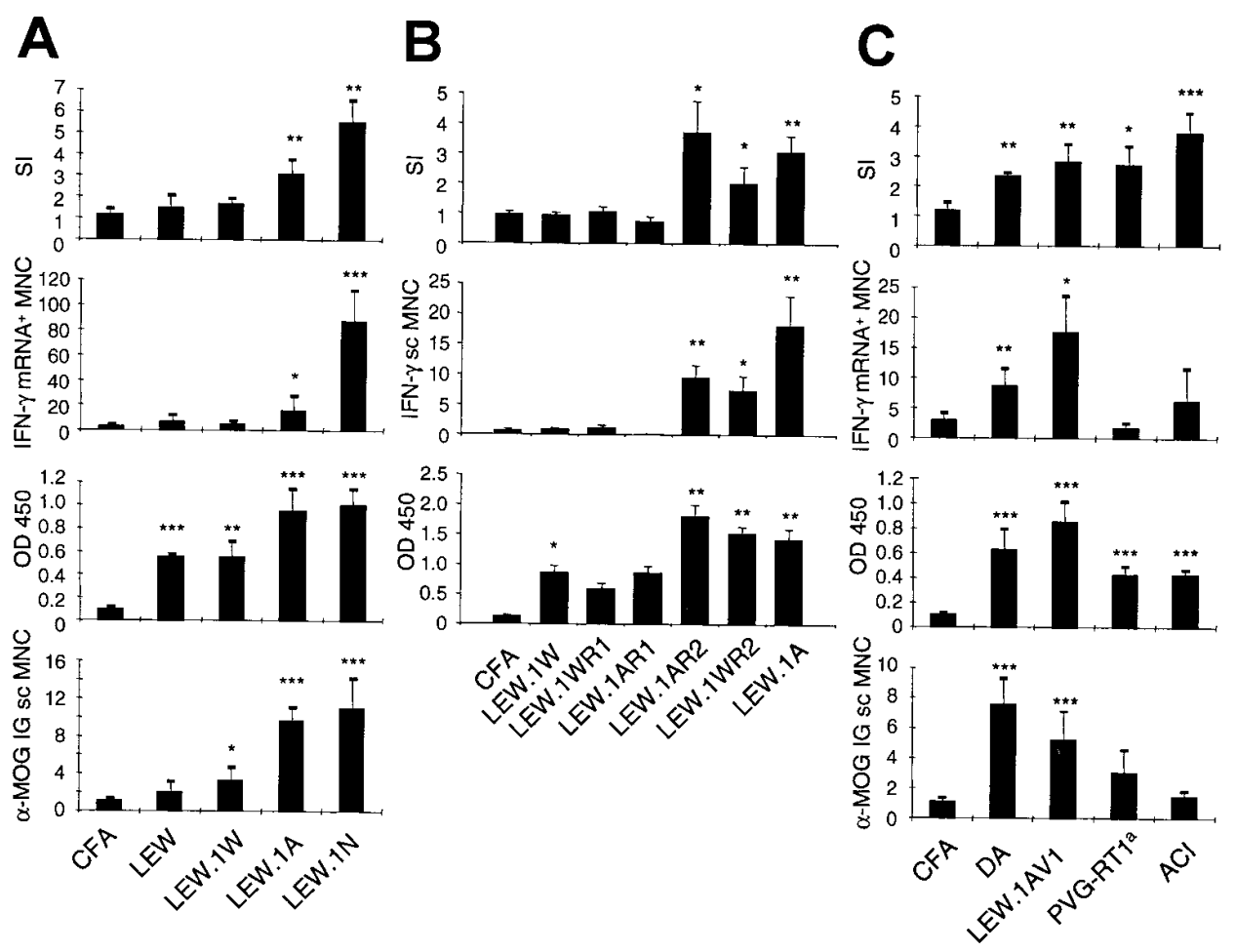

Figure 3. MOG-specific T and $\mathrm{B}$ cell responses at day 12 p.i. $(A)$ MOG-immunized LEW $(n=8)$, LEW.1W $(n=8)$, LEW.1A $(n=8)$, LEW.1N $(n=8)$ rats, and CFAimmunized rats $(n=10) ;(B)$ MOGimmunized LEW.1W $(n=6)$, LEW.1AR1 $(n=5)$, LEW.1WR1 $(n=6)$, LEW.1A $(n=5)$, LEW.1AR2 $(n=5)$, LEW.1WR2 $(n=6)$, and CFA-immunized rats $(n=6) ;(C)$ MOG-immunized DA $(n=9)$, LEW.1AV1 $(n=6)$, PVG$\mathrm{RT}^{\mathrm{a}}(n=8)$, ACI $(n=7)$ rats, and CFA-immunized rats $(n=10)$. SI, proliferative $\mathrm{T}$ cell responses from draining LN; IFN- $\gamma$ mRNA ${ }^{+}$MNC, numbers of Ag-specific IFN- $\gamma$ mRNA-expressing LN cells; IFN- $\gamma$ sc MNC, numbers of Ag-specific IFN- $\gamma$-secreting LN cells; OD 450, specific anti-MOG IgG levels in serum; $\alpha$-MOG IG sc MNC, specific anti-MOG Ab-secreting cells per $10^{5} \mathrm{LN}$ cells. Each bar represents mean \pm SEM. Stars indicate significance of responses in MOG-immunized rats compared with CFAimmunized rats $(* P<0.05$;

**P $P<0.01$;***P<0.001). After normality testing with the Shapiro-Wilks test, $P$ values were calculated with the Student's $t$ test in cases of normal distribution, otherwise the Mann-Whitney U test was used. 
A
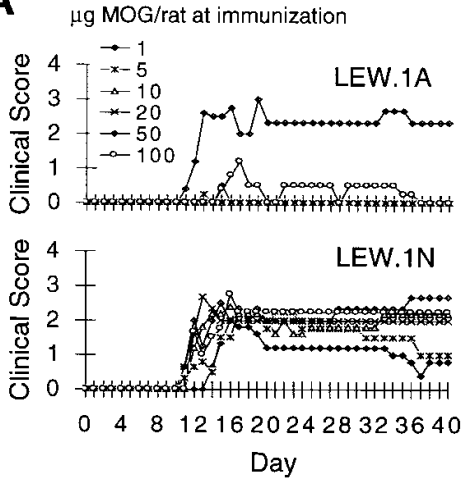

B
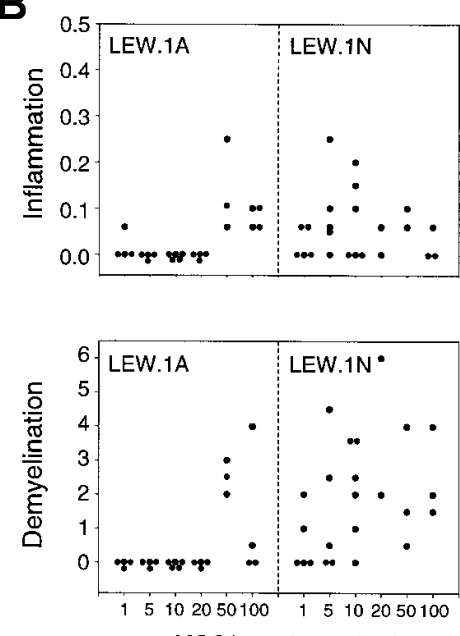

C $\mu \mathrm{g} \mathrm{MOG} /$ rat at immunization

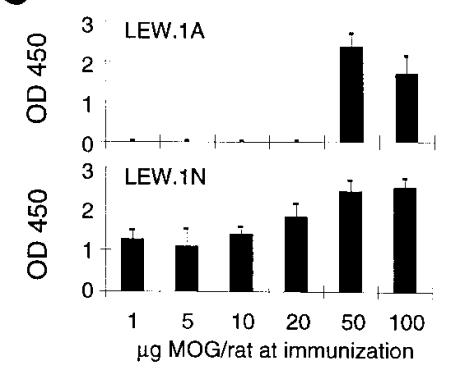

Figure 4. Titration of $\mathrm{Ag}$ for disease triggering in LEW.1A and LEW.1N rats. Groups of 5-6 LEW.1N and 4-5 LEW.1A rats were immunized with $1,5,10$, 20,50 , or $100 \mu \mathrm{g}$ of MOG in CFA. (A) Mean daily clinical scores (in the LEW.1N groups immunized with 100,50 , and $20 \mu \mathrm{g}$ of MOG 2 of 5, 4 of 6 , and 4 of 6 rats died between days 12-14 p.i., respectively, and in the LEW.1A groups immunized with 100 and 50 $\mu \mathrm{g}$ MOG, 1 of 5 and 2 of 5 rats died between days 13-17 p.i., respectively); (B) CNS inflammation and demyelination on day 40 p.i.; $(C)$ MOG-specific serum Ab titers day 12 p.i. LEW.1N rats developed disease at all tested Ag doses, while LEW.1A rats developed disease only at doses $>20 \mu \mathrm{g}$ of MOG. Disease was paralleled by MOG-specific serum $\mathrm{Ab}$.

doses of autoantigen. Once again, the induction of clinical disease correlated with the MOG-specific immune response (Fig. $4 C$ ). LEW.1N rats displayed high MOG-specific Ab titers at all doses tested, while LEW.1A rats raised MOG-specific Ab only at doses of 50 or $100 \mu \mathrm{g}$. Thus, the identity of the MHC also determined the amount of Ag necessary to trigger autoimmune disease.

RT1.B/D allelic products are the key regulators of susceptibility in MOG-EAE. To narrow the regions within the MHC that modulated the disease phenotypes, we used different combinations of RT1.A/B/D/C alleles of the $\mathrm{RT} 1^{\mathrm{a}}$ and $\mathrm{RT} 1^{\mathrm{u}}$ haplotype on the permissive LEW background $(10,15)$ (Table I). IntraMHC recombinant rats with recombination events between RT1.A and RT1.B (LEW.1AR1 and LEW.1WR2, respectively) and RT1.D and RT1.C (LEW.1AR2 and LEW.1WR1, respectively) allowed us to map the region within the MHC and determine susceptibility to MOG-induced EAE. The clinical dis-

Table I. Inbred Rat Strain Designations with Fine Mapping and Recombination Sites of the Rat MHC (RT1)

\begin{tabular}{|c|c|c|c|c|c|c|}
\hline \multirow[b]{2}{*}{ Strain } & \multirow[b]{2}{*}{ Haplotype } & \multirow{2}{*}{$\begin{array}{l}\text { Class I } \\
\text { RT1.A }\end{array}$} & \multicolumn{2}{|c|}{ Class II } & \multirow[b]{2}{*}{ Class III } & \multirow{2}{*}{$\begin{array}{l}\text { Class I } \\
\text { RT1.C }\end{array}$} \\
\hline & & & RT1.B & RT1.D & & \\
\hline DA & av1 & $\mathrm{a}$ & $\mathrm{a}$ & a & av1 & av1 \\
\hline ACI & av1 & $\mathrm{a}$ & $\mathrm{a}$ & a & av1 & av1 \\
\hline PVG-RT1ª (DA) & av1 & $\mathrm{a}$ & $\mathrm{a}$ & $\mathrm{a}$ & av1 & av1 \\
\hline LEW.1AV1 (DA) & av1 & a & $\mathrm{a}$ & $\mathrm{a}$ & av1 & av1 \\
\hline LEW.1A (AVN) & $\mathrm{a}$ & $\mathrm{a}$ & $\mathrm{a}$ & a & $\mathrm{a}$ & a \\
\hline LEW.1N (BN) & $\mathrm{n}$ & $\mathrm{n}$ & $\mathrm{n}$ & $\mathrm{n}$ & $\mathrm{n}$ & $\mathrm{n}$ \\
\hline LEW.1W (WP) & $\mathrm{u}$ & $\mathrm{u}$ & $\mathrm{u}$ & $\mathrm{u}$ & $\mathrm{u}$ & $\mathrm{u}$ \\
\hline LEW & 1 & 1 & 1 & 1 & 1 & 1 \\
\hline LEW.1AR1 & r2 & $\mathrm{a}$ & $\mathrm{u}$ & $\mathrm{u}$ & $\mathrm{u}$ & $\mathrm{u}$ \\
\hline LEW.1AR2 & r3 & $\mathrm{a}$ & $\mathrm{a}$ & a & $\mathrm{u}$ & $\mathrm{u}$ \\
\hline LEW.1WR1 & $\mathrm{r} 4$ & $\mathrm{u}$ & $\mathrm{u}$ & $\mathrm{u}$ & $\mathrm{a}$ & $\mathrm{a}$ \\
\hline LEW.1WR2 & r6 & $\mathrm{u}$ & $\mathrm{a}$ & $\mathrm{a}$ & $\mathrm{a}$ & $\mathrm{a}$ \\
\hline DA.1H & $\mathrm{h}$ & $\mathrm{h}$ & $\mathrm{n}$ & $\mathrm{n}$ & $?$ & $?$ \\
\hline $\mathrm{BN}$ & $\mathrm{n}$ & $\mathrm{n}$ & $\mathrm{n}$ & $\mathrm{n}$ & $\mathrm{n}$ & $\mathrm{n}$ \\
\hline
\end{tabular}

Donor strain in parentheses.

ease courses are outlined in Fig. $1 B$, and the histopathological evaluation is given in Fig. 2.

Most LEW.1AR2 $\left(\mathrm{RT}^{\mathrm{r} 3}\right)$ rats that are RT1.A $\mathrm{a} / \mathrm{B}^{\mathrm{a}} / \mathrm{D}^{\mathrm{a}}$ and RT1.C $^{\mathrm{u}}(17 / 18)$ and most LEW.1WR2 $\left(\mathrm{RT}^{\mathrm{r} 6}\right)$ rats that are RT1.A ${ }^{\mathrm{u}}$ and RT1.B ${ }^{\mathrm{a}} / \mathrm{C}^{\mathrm{a}} / \mathrm{D}^{\mathrm{a}}$ (12/13) developed early disease, sharing the MHC class II RT1.B $/ \mathrm{D}^{\mathrm{a}}$ alleles of the LEW.1A rat (Table I). Only a low number of LEW.1AR1 $\left(\mathrm{RT}^{\mathrm{r} 2}\right)$ rats that are RT1. $A^{\mathrm{a}}$ and $\mathrm{RT} 1 . \mathrm{B}^{\mathrm{u}} / \mathrm{D}^{\mathrm{u}} / \mathrm{C}^{\mathrm{u}}$ developed clinical disease (7/ 27). This was also the case for LEW.1WR1 $\left(\mathrm{RT}^{\mathrm{r} 4}\right)$ rats with RT1.A ${ }^{\mathrm{u}} / \mathrm{B}^{\mathrm{u}} / \mathrm{D}^{\mathrm{u}}$ and RT1.C $\mathrm{C}^{\mathrm{a}}(7 / 28)$. Both strains share the $\mathrm{RT} 1 . \mathrm{B}^{\mathrm{u}} / \mathrm{D}^{\mathrm{u}}$ alleles of the LEW.1W rat in the MHC class II region.

Consistent with the early onset of clinical disease in LEW.1AR2 rats, at day 12 p.i., two-thirds of the rats had inflammation alone or inflammation associated with demyelination, while by day 40 p.i., most animals had demyelinating lesions in the spinal cord and the brain. A number of LEW.1WR2 rats showed inflammation on day 12 p.i. that was not associated with demyelination, but large confluent plaques of demyelination were observed by day 40 p.i., predominantly in the spinal cord (Fig. 5). LEW.1WR1 and LEW.1AR1 rats had no or minimal inflammation and no demyelination in the CNS by day 12 p.i. As with the LEW.1W strain, some rats from these two strains developed demyelinated lesions and clinical symptoms late in the observation period. The LEW.1AR1 strain was especially prone to develop CNS lesions, as discussed below.

T cell proliferation assays, Elispot assays to assess the number of IFN- $\gamma$-secreting cells, and serum titers of anti-MOG Ab on day 12 p.i. revealed that rats having the $\mathrm{RT} 1 . \mathrm{B}^{\mathrm{a}} / \mathrm{D}^{\mathrm{a}}$ alleles (LEW.1A, LEW.1AR2, and LEW.1WR2) had higher MOGspecific immune responses than rats having the $\mathrm{RT} 1 . \mathrm{B}^{\mathrm{u}} / \mathrm{D}^{\mathrm{u}}$ alleles (LEW.1W, LEW.1AR1, and LEW.1WR1) (Fig. 3 B). All strains had high proliferation indices and between 500 and 2,000 IFN- $\gamma$-secreting cells per $4 \times 10^{5}$ cells after stimulation with ConA.

The main susceptibility locus within the MHC for MOGinduced EAE thus maps to the RT1.B/D region. This was 

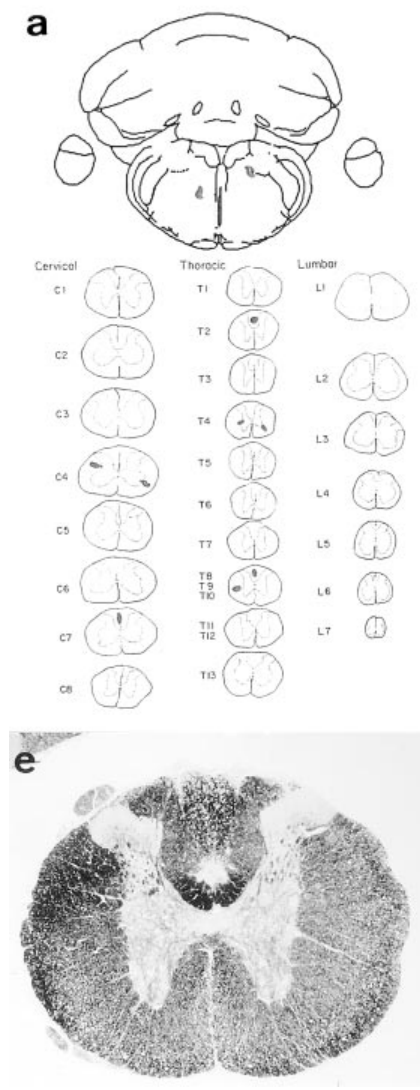

b
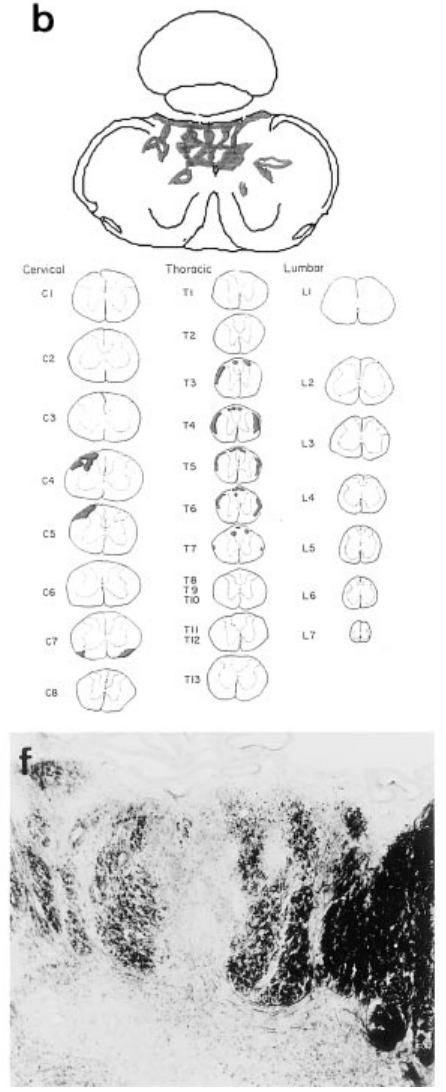

C

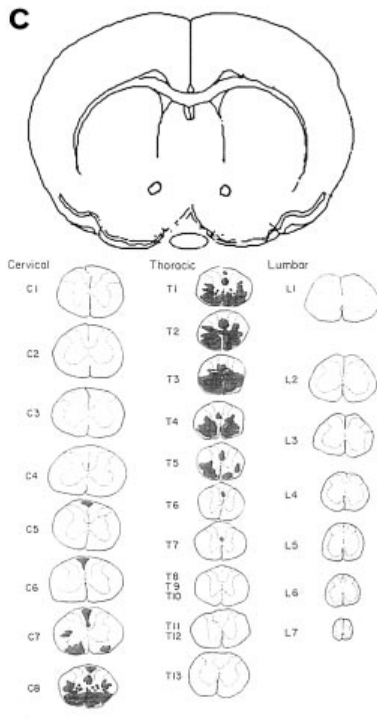

g

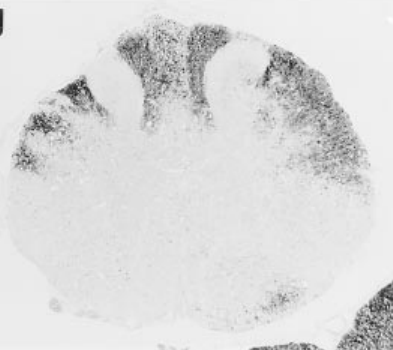

d

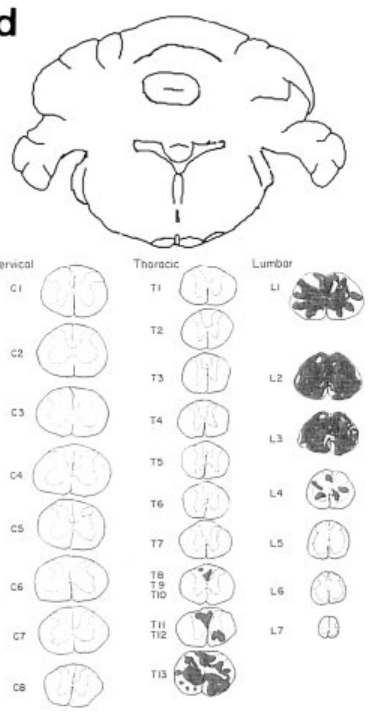

h

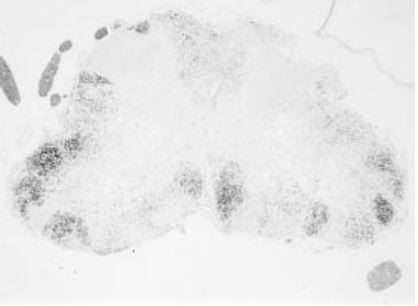

Figure 5. Histopathology of different RT1 congenic rat strains after immunization with MOG. Comparison of lesional topography and lesion formation of actively induced MOG-EAE is illustrated for LEW $(a, e)$, LEW.1N $(b, f)$, LEW.1A $(c, g)$, and LEW.1WR2 $(d, h)$ rats. $e-h$ illustrate the degree of demyelination (Luxol fast blue myelin stain). $a, e$ : LEW rats show small perivascular rims of demyelination $(e \times 27) . b, f: \mathrm{LEW} .1 \mathrm{~N}$ rats exhibit widespread demyelination oriented to the CSF and in association with the area postrema $(f \times 70)$. $c, g$ : LEW.1A rats show focal confluent demyelination in the spinal cord $(g \times 27) . d, h$ : LEW.1WR2 rats frequently present transverse myelitis $(h \times 25)$.

strengthened by the observation that four out of four investigated DA.1H $\left(\mathrm{RT}^{\mathrm{h}}\right)$ rats, which carry the RT1.B $/ \mathrm{D}^{\mathrm{n}}$ alleles in the MHC class II but different MHC class I and III alleles (Table I), developed an acute and lethal disease similar to the LEW.1N rat strain (data not shown). Further support comes from the observation that $\mathrm{BN}$ rats, which are commonly considered to be resistant to EAE-induction (18) but which are also RT1.B ${ }^{\mathrm{n}} / \mathrm{D}^{\mathrm{n}}$ (Table I), are highly susceptible to MOGinduced EAE (Linington, C., Weissert, R., unpublished observation). To rule out possible influences from genetic drift of the LEW.1N strain, intercross F2(LEW.1N $\times$ LEW) rats were investigated to confirm linkage of acute lethal disease to RT1 ${ }^{\mathrm{n}}$ (data not shown).

An influence of the RT1.A region (MHC class I) on lesion development in MOG-induced EAE was suggested by the more pronounced demyelination in the brain and spinal cord in the LEW.1AR1 $\left(\mathrm{RT}^{\mathrm{r}}{ }^{2}\right)$ compared with the LEW.1W $\left(\mathrm{RT}^{\mathrm{u}}\right)$ strain on day 40 p.i. (Fig. 2). Due to the recombination between RT1.A ${ }^{\mathrm{a}}$ and RT1.B ${ }^{\mathrm{u}} / \mathrm{D}^{\mathrm{u}} / \mathrm{C}^{\mathrm{u}}\left(\mathrm{RT}^{\mathrm{r}} \mathrm{2}\right)$ in the LEW.1AR1 strain (Table I), this difference could map to RT1. $\mathrm{A}^{\mathrm{a}}$ or genes downstream within the MHC congene fragment, or an interaction between RT1. $\mathrm{A}^{\mathrm{a}}$ and $\mathrm{RT} 1 . \mathrm{B}^{\mathrm{u}} / \mathrm{D}^{\mathrm{u}} / \mathrm{C}^{\mathrm{u}}$, as discussed below. Additional disease-modifying influences mediated by RT1.C, MHC class III region genes, or loci located further downstream were suggested by the comparison of the disease course between the LEW.1AV1 and LEW.1A rats, which differ in the
RT1 downstream of the RT1.D gene region (Table I). While none out of ten LEW.1A rats had a relapsing/remitting type of disease, but instead developed chronic neurological deficits, five out of ten LEW.1AV1 rats developed a relapsing remitting disease course $(P=0.02$; Fisher's exact test).

Modulation of $R T 1^{a v 1}$ influences by four different nonMHC backgrounds. We investigated the degree of susceptibility, clinical disease course, and lesional pathology in four dif-

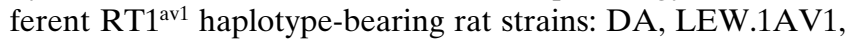
PVG-RT1 ${ }^{a}$, and ACI (Figs. $1 C$ and 2). Five out of nine DA rats and five out of ten LEW.1AV1 rats exhibited a relapsing/ remitting disease course with classical ascending paraparesis. In addition, two out of ten LEW.1AV1 rats displayed ataxia (data not shown). Thus, the RT1 $1^{\text {av1 }}$ haplotype permitted a similar clinical disease phenotype on the LEW and DA rat non-MHC genes, with slightly more acute symptoms in the LEW.1AV1 rat. In contrast, the PVG and ACI non-MHC genes were less permissive for MOG-induced EAE in conjunction with the $\mathrm{RT}^{\text {av1 }}$ haplotype.

At day 12 p.i., PVG-RT1 ${ }^{\mathrm{a}}$ and ACI rats displayed little or no CNS pathology. In contrast, LEW.1AV1 and DA rats had already developed extensive CNS lesions by day 12 p.i. In both strains, large plaques of demyelination with variable demyelinating activity were found in both the brain and spinal cord by day 40 p.i. Actively demyelinating areas were characterized by infiltration with $\mathrm{T}$ cells and macrophages, and destruction of 
myelin was associated with the deposition of $\mathrm{C} 9$ and $\mathrm{IgG}$. In inactive areas, the inflammatory lesions contained numerous macrophages. Gliotic scar formation was evident in areas with confluent demyelination and axonal density was only slightly reduced. Thus, the $\mathrm{RT} 1^{\text {av1 }}$ haplotype on two different EAEpermissive non-MHC backgrounds resulted in similar histopathological changes that were strongly reminiscent of those seen in MS.

To study if the regulation of disease outcome by interactions involving the MHC haplotype and non-MHC genes was related to any qualitative or quantitative feature of the autoimmune response to MOG, we analyzed MOG-specific T and B cell responses in the four strains with the $\mathrm{RT}^{\text {av1 }}$ haplotype on day 12 p.i. (Fig. $3 C$ ). MOG induced a proliferative response in the LN cell cultures of all four rat strains on day 12 p.i. However, an increase in numbers of IFN- $\gamma$ mRNA-expressing cells was recorded only in the disease-susceptible LEW.1AV1 and DA rat strains (Fig. $3 \mathrm{C}$ ), although all four strains displayed similar increases after mitogenic stimulation with Con A (data not shown). The numbers of anti-MOG Ab-secreting cells were also higher in DA rats and LEW.1AV1 rats than in PVG$\mathrm{RT}^{\mathrm{a}}$ rats and ACI rats. This correlated with the anti-MOG $\mathrm{Ab}$ titers. Thus, the $\mathrm{RT} 1^{\mathrm{av} 1}$ haplotype is permissive for induction of a MOG-specific autoimmune response in all strains, but the functional maturation of this response is strongly influenced by non-MHC genes.

\section{Discussion}

We demonstrate that MHC haplotypes differ in the severity of the ensuing disease they permit in response to a constant autoantigenic MOG challenge. Furthermore, the MHC haplotype determines the amount of autoantigen needed to induce disease. Thus, MHC haplotypes are not strictly disease permissive or resistant. Instead, there is a hierarchy in MHC haplotype regulation of disease, with high responder haplotypes such as $\mathrm{RT}^{\mathrm{n}}$ and $\mathrm{RT} 1^{\mathrm{h}}$, intermediate ones such as $\mathrm{RT} 1^{\mathrm{a}}$, $\mathrm{RT}^{\mathrm{av} 1}, \mathrm{RT}^{\mathrm{r} 3}$, and $\mathrm{RT} 1^{\mathrm{r} 6}$, and low responders such as $\mathrm{RT}^{\mathrm{u}}$, $\mathrm{RT}^{\mathrm{r} 2}, \mathrm{RT}^{\mathrm{r} 4}$, and RT1 ${ }^{1}$. Notably, no single haplotype is completely protected (19). This form of MHC haplotype-regulated graded influence on MOG-EAE, a model that is more like MS than any other EAE model (8, 9, the present work), gives perspective on how HLA haplotypes might be involved in the regulation of human MS. Similar to what is observed here, graded influences are apparent. The HLA-DR2(15) haplotype strikingly increases the risk to develop MS, followed by HLADR3(17), while a series of other HLA haplotypes also permit disease (20). Hypothetically, and consistent with the present observations in MOG-EAE, the former haplotypes would allow disease with milder environmental triggers and/or a less susceptible non-MHC background genome, and vice versa, with regard to less permissive HLA haplotypes. Since neither the triggering events nor the background genomes can be experimentally manipulated in humans, we believe that our findings in MOG-induced EAE are important as a basis for further mechanistic studies to understand how the MHC regulates complex organ-specific inflammatory diseases. At present, we hypothesize that allelic variations in the MHC class II molecule peptide binding abilities might be responsible for the dramatic differences between the different haplotypes in response to immunization with MOG. The antigen-MHC interaction decisively influences the developing $\mathrm{T}$ cell repertoire within the thymus (21-24) and the degree and quality of peripheral $\mathrm{T}$ cell activation $(25,26)$. T cell transfer experiments show that the numbers and functional differentiation of adoptively transferred $\mathrm{T}$ cells correlate with the severity of the subsequent disease (27-29). We currently explore how allelic variations in the MHC class II molecule binding abilities to MOG peptides correlate to the selection, expansion, and epitope specificity of encephalitogenic T and B cells. This study strongly suggests that the mechanism for $\mathrm{MHC}$ haplotype regulation of the degree of ensuing disease is by regulation of the quantity and quality of the autoimmune response after autoantigenic challenge, since these factors directly correlated to each other.

The use of intra-MHC recombinant rats mapped major regulatory influences to the $\mathrm{MHC}$ class II region. A strong argument in favor of a role for RT1.B and/or RT1.D molecules (equivalent to human HLA-DQ and HLA-DR, respectively) is the different MHC haplotype restriction of MOG-induced disease $(19,30$, the present work) as compared with MBPinduced EAE in LEW compared with LEW.1N rats (31, Table II). This is difficult to reconcile with any general immunoregulatory genes in the MHC class II region, since only allelic forms of the RT.1B and RT.1D molecules would discriminate between Ags. Interestingly, the RT1 ${ }^{\text {av1 }}$ haplotype is highly disease permissive to MBP, PLP, and MOG as well (32-34, the present work). By this ability, $\mathrm{RT}^{\text {av1 }}$ is CNS-autoantigen promiscuous, unlike the $\mathrm{RT}^{\mathrm{n}}$ haplotype. Since MHC class II molecules are restriction elements for $\mathrm{CD}^{+}{ }^{+} \mathrm{T}$ cells, another argument supporting the major role of the RT1.B and RT1.D is the correlation between the severity of clinical disease and the magnitude of the MOG-specific T cell response. MOG-specific $\mathrm{B}$ cell responses also show a correlation to disease severity, a phenomenon that we regard as probably secondary to the $\mathrm{T}$ cell response (35-37). The degree of the ensuing B cell response might well be causally related to the degree of ensuing disease in view of the importance of anti-MOG antibodies in demyelination and the potential antigen-presenting ability of B cells (38). Apart from the classical MHC class II molecules themselves, some other immunoregulatory genes also map within the MHC class II region. An important example of this is RT1.DM (the equivalent of the human HLA-DM) (39), and a final proof for a particular gene being instrumental may necessitate its knockout and/or transgenic expression. Furthermore, there are haplotype-specific disease-promoting influences from genes down- or upstream from the MHC class II region. Recombinations between the MHC class I and class II region showed that the RT1. $A^{a}$ allele in conjunction with the $\mathrm{RT} 1 . \mathrm{B}^{\mathrm{u}} / \mathrm{D}^{\mathrm{u}}\left(\mathrm{RT}^{\mathrm{r}}\right)$ alleles resulted in conspicuous demyelination late in disease compared with rats with $\mathrm{RT} 1 . \mathrm{A}^{\mathrm{u}} / \mathrm{B}^{\mathrm{u}} / \mathrm{D}^{\mathrm{u}}$

Table II. Autoantigen Restrictions in Rat EAE

\begin{tabular}{lcccc}
\hline & \multicolumn{4}{c}{ RT1 Haplotype } \\
\cline { 2 - 5 } Autoantigen & RT1 $^{1}$ & RT1 $^{\mathrm{u}}$ & $\mathrm{RT}^{\mathrm{a}}$ RT1 $^{\mathrm{av} 1}$ & $\mathrm{RT}^{\mathrm{n}}$ \\
\hline MBP & $+++(31)$ & ND & $+++(32,34)$ & $-(31)$ \\
PLP & $+(33)$ & ND & $+++(34)$ & ND \\
MOG & $(+)^{*}(19,30)$ & $+*$ & $+++*$ & $+++*$ \\
& & & & \\
\hline
\end{tabular}

*The present work. 
$\left(\mathrm{RT} 1^{\mathrm{u}}\right)$ alleles. The mechanism responsible for this effect is unclear and is under study in our laboratories. Besides effects from the MHC class I region itself, this might depend on the TAP2 genes that exhibit allelic polymorphisms between LEW.1W (TAP2 $^{\mathrm{b}}$ ), LEW.1A (TAP2 ${ }^{\mathrm{a}}$ ), and LEW.1AR1 (TAP2 ${ }^{\mathrm{b}}$ ) rats, which results in a differential loading of peptides onto class I MHC molecules (cim effect) (40). Genes upstream from the MHC class II region in the RT $1^{\text {av1 }}$ haplotype might also modulate clinical disease type since LEW.1AV1, but not LEW.1A, rats developed a relapsing/remitting disease course.

Non-MHC genes could abrogate MHC-permitted susceptibility to MOG-induced EAE. By changing non-MHC genes on a constant MHC haplotype, we show that non-MHC genes are disease protective in PVG-RT1 $1^{\mathrm{a}}$ and $\mathrm{ACI}$, and permissive in DA and LEW.AV1 rats. Interestingly, while all strains mounted a marked proliferative response to MOG in vitro, only the disease-permissive ones displayed MOG-induced IFN- $\gamma$ mRNA expression and a strong MOG-specific B cell response. This demonstrates that genes outside the MHC determine the pathogenic potential of $\mathrm{T}$ cells by regulating their functional differentiation, for instance, at the level of IFN- $\gamma$ expression and secretion, or providing B cell help. Findings consistent with this have been reported in mice (41). Furthermore, in MBP-induced EAE, exogenous IL-12 was necessary for disease induction in B10.S mice (42). We are currently mapping the non-MHC genes that modulate RT1 mediated effects in MOG-EAE by genome-wide microsatellite screening (43) of an F2 intercross between DA and ACI rats.

In conclusion, we have explored the immunogenetic characteristics of the new and very MS-like MOG-EAE in rats. This gives an experimental basis for a hypothetical model of MHC influences in complex organ-specific inflammatory diseases, in which an MHC-haplotype-dependent hierarchically ordered susceptibility is modulated by environmental triggers and other, still undefined, non-MHC background genes.

\section{Acknowledgments}

We thank M. Khademi for technical assistance with in situ hybridizations, K. L. de Graaf for help with cell preparations, and E. Berg (Unit of Medical Statistics, Karolinska Institute, Sweden) for statistical advice.

This study was supported by the European Union (Biomed 2, Contract No. BMH4-97-2027), the Deutsche Forschungsgemeinschaft (SFB 217, Projekt C14), the Swedish Medical Research Council, the Swedish Society for the Neurologically Disabled, the Petrus and Augusta Hedlunds Stiftelse, and the AFA Foundation. C. Linington holds a Hermann-Lilly-Schilling Professorship. R. Weissert is a postdoctoral fellow of the Deutsche Forschungsgemeinschaft (We 1947/1-1).

\section{References}

1. Vyse, T.J., and J.A. Todd. 1996. Genetic analysis of autoimmune disease. Cell. 85:311-318.

2. Olerup, O., and J. Hillert. 1991. HLA class II-associated genetic susceptibility in multiple sclerosis: a critical evaluation. Tissue Antigens. 38:1-15.

3. Ebers, G.C., and A.D. Sadovnick. 1998. Chapter 3. In Multiple Sclerosis. D.W. Paty and G.C. Ebers, editors. F.A. Davis Co., Philadelphia. 29-47.

4. Wekerle, H., K. Kojima, J. Lannes-Vieira, H. Lassmann, and C. Linington. 1994. Animal models. Ann. Neurol. 36(Suppl.):47-53.

5. Steinman, L. 1996. Multiple sclerosis: a coordinated immunological attack against myelin in the central nervous system. Cell. 85:299-302.

6. Tisch, R., and H. McDevitt. 1996. Insulin-dependent diabetes mellitus. Cell. 85:291-297.

7. Lucchinetti, C.F., W. Bruck, M. Rodriguez, and H. Lassmann. 1996. Dis- tinct patterns of multiple sclerosis pathology indicates heterogeneity in pathogenesis. Brain Pathol. 6:259-274.

8. Genain, C.P., M.H. Nguyen, N.L. Letvin, R. Pearl, R.L. Davis, M. Adelman, M.B. Lees, C. Linington, and S.L. Hauser. 1995. Antibody facilitation of multiple sclerosis-like lesions in a nonhuman primate. J. Clin. Invest. 96:29662974.

9. Linington, C., M. Bradl, H. Lassmann, C. Brunner, and K. Vass. 1988. Augmentation of demyelination in rat acute allergic encephalomyelitis by circulating mouse monoclonal antibodies directed against a myelin/oligodendrocyte glycoprotein. Am. J. Pathol. 130:443-454.

10. Hedrich, H.J. 1990. Genetic Monitoring of Inbred Strains of Rats. Gustav Fischer Verlag, New York. 539 pp.

11. Kojima, K., T. Berger, H. Lassmann, D. Hinze-Selch, Y. Zhang, J. Gehrmann, K. Reske, H. Wekerle, and C. Linington. 1994. Experimental autoimmune panencephalitis and uveoretinitis transferred to the Lewis rat by $\mathrm{T}$ lymphocytes specific for the $\mathrm{S} 100$ beta molecule, a calcium binding protein of astroglia. J. Exp. Med. 180:817-829.

12. Amor, S., N. Groome, C. Linington, M.M. Morris, K. Dornmair, M.V. Gardinier, J.M. Matthieu, and D. Baker. 1994. Identification of epitopes of myelin oligodendrocyte glycoprotein for the induction of experimental allergic encephalomyelitis in SJL and Biozzi AB/H mice. J. Immunol. 153:4349-4356.

13. Piddlesden, S.J., M.K. Storch, M. Hibbs, A.M. Freeman, H. Lassmann, and B.P. Morgan. 1994. Soluble recombinant complement receptor 1 inhibits inflammation and demyelination in antibody-mediated demyelinating experimental allergic encephalomyelitis. J. Immunol. 152:5477-5484.

14. Vass, K., H. Lassmann, H. Wekerle, and H.M. Wisniewski. 1986. The distribution of Ia antigen in the lesions of rat acute experimental allergic encephalomyelitis. Acta Neuropathol. 70:149-160.

15. Mustafa, M., C. Vingsbo, T. Olsson, S. Issazadeh, A. Ljungdahl, and R. Holmdahl. 1994. Protective influences on experimental autoimmune encephalomyelitis by MHC class I and class II alleles. J. Immunol. 153:3337-3344.

16. Zachau, A.C., K. Strigard, S. Baig, B. Hojeberg, and T. Olsson. 1989. Distribution of plasma cells secreting antibodies against nervous tissue antigens during experimental allergic encephalomyelitis enumerated by a nitrocellulose immunospot assay. J. Neurol. Sci. 91:323-336.

17. Wedekind, D., C.M. Prokop, U. Brunsberg, R. Holmdahl, and H.J. Hedrich. 1997. Identification and differentiation of transcribed major histocompatibility complex class II RT1B alpha and RT1B beta alleles from laboratory rats. Transplant. Proc. 29:1668-1669.

18. Levine, S., and R. Sowinski. 1975. Allergic encephalomyelitis in the reputedly resistant Brown Norway strain of rats. J. Immunol. 114:597-601.

19. Johns, T.G., N. Kerlero de Rosbo, K.K. Menon, S. Abo, M.F. Gonzales, and C.C. Bernard. 1995. Myelin oligodendrocyte glycoprotein induces a demyelinating encephalomyelitis resembling multiple sclerosis. J. Immunol. 154: $5536-5541$.

20. Hillert, J. 1996. Genetics of multiple sclerosis. In Handbook of Multiple Sclerosis. S.D. Cook, editor. Marcel Dekker Inc., New York. 19-51.

21. Blackman, M.A., P. Marrack, and J. Kappler. 1989. Influence of the major histocompatibility complex on positive thymic selection of $\mathrm{V}$ beta $17 \mathrm{a}+\mathrm{T}$ cells. Science. 244:214-217.

22. Sebzda, E., V.A. Wallace, J. Mayer, R.S. Yeung, T.W. Mak, and P.S. Ohashi. 1994. Positive and negative thymocyte selection induced by different concentrations of a single peptide. Science. 263:1615-1618.

23. Nakano, N., R. Rooke, C. Benoist, and D. Mathis. 1997. Positive selection of T cells induced by viral delivery of neopeptides to the thymus. Science. 275:678-683.

24. Schmidt, D., J. Verdaguer, N. Averill, and P. Santamaria. 1997. A mechanism for the major histocompatibility complex-linked resistance to autoimmunity. J. Exp. Med. 186:1059-1075.

25. Hammer, J., F. Gallazzi, E. Bono, R.W. Karr, J. Guenot, P. Valsasnini, Z.A. Nagy, and F. Sinigaglia. 1995. Peptide binding specificity of HLA-DR4 molecules: correlation with rheumatoid arthritis association. J. Exp. Med. 181: 1847-1855.

26. Pfeiffer, C., J. Stein, S. Southwood, H. Ketelaar, A. Sette, and K. Bottomly. 1995. Altered peptide ligands can control CD4 T lymphocyte differentiation in vivo. J. Exp. Med. 181:1569-1574.

27. Lassmann, H., C. Brunner, M. Bradl, and C. Linington. 1988. Experimental allergic encephalomyelitis: the balance between encephalitogenic $\mathrm{T}$ lymphocytes and demyelinating antibodies determines size and structure of demyelinated lesions. Acta Neuropathol. 75:566-576.

28. Baron, J.L., J.A. Madri, N.H. Ruddle, G. Hashim, and C.A. Janeway, Jr. 1993. Surface expression of alpha 4 integrin by CD4 T cells is required for their entry into brain parenchyma. J. Exp. Med. 177:57-68.

29. Berger, T., S. Weerth, K. Kojima, C. Linington, H. Wekerle, and H. Lassmann. 1997. Experimental autoimmune encephalomyelitis: the antigen specificity of T lymphocytes determines the topography of lesions in the central and peripheral nervous system. Lab. Invest. 76:355-364.

30. Adelmann, M., J. Wood, I. Benzel, P. Fiori, H. Lassmann, J.M. Matthieu, M.V. Gardinier, K. Dornmair, and C. Linington. 1995. The N-terminal domain of the myelin oligodendrocyte glycoprotein (MOG) induces acute demyelinating experimental autoimmune encephalomyelitis in the Lewis rat. $J$. Neuroimmunol. 63:17-27. 
31. Happ, M.P., P. Wettstein, B. Dietzschold, and E. Heber-Katz. 1988. Genetic control of the development of experimental allergic encephalomyelitis in rats. Separation of MHC and non-MHC gene effects. J. Immunol. 141:14891494.

32. Lorentzen, J.C., S. Issazadeh, M. Storch, M.I. Mustafa, H. Lassman, C. Linington, L. Klareskog, and T. Olsson. 1995. Protracted, relapsing and demyelinating experimental autoimmune encephalomyelitis in DA rats immunized with syngeneic spinal cord and incomplete Freunds adjuvant. J. Neuroimmunol. 63:193-205.

33. Yamamura, T., T. Namikawa, M. Endoh, T. Kunishita, and T. Tabira. 1986. Experimental allergic encephalomyelitis induced by proteolipid apoprotein in Lewis rats. J. Neuroimmunol. 12:143-153.

34. Stepaniak, J.A., K.E. Gould, D. Sun, and R.H. Swanborg. 1995. A comparative study of experimental autoimmune encephalomyelitis in Lewis and DA rats. J. Immunol. 155:2762-2769.

35. Jones, B., and C.A. Janeway, Jr. 1981. Cooperative interaction of B lymphocytes with antigen-specific helper T lymphocytes is MHC restricted. Nature. 292:547-549.

36. Lanzavecchia, A. 1985. Antigen-specific interaction between T and B cells. Nature. 314:537-539.

37. Parker, D.C. 1993. T cell-dependent B cell activation. Annu. Rev. Im- munol. 11:331-360.

38. Lanzavecchia, A. 1990. Receptor-mediated antigen uptake and its effect on antigen presentation to class II-restricted T lymphocytes. Annu. Rev. Immunol. 8:773-793.

39. Kropshofer, H., G.J. Hammerling, and A.B. Vogt. 1997. How HLA-DM edits the MHC class II peptide repertoire: survival of the fittest? Immunol. Today. 18:77-82

40. Powis, S.J., L.L. Young, E. Joly, P.J. Barker, L. Richardson, R.P Brandt, C.J. Melief, J.C. Howard, and G.W. Butcher. 1996. The rat cim effect: TAP allele-dependent changes in a class I MHC anchor motif and evidence against C-terminal trimming of peptides in the ER. Immunity. 4:159-165.

41. Gorham, J.D., M.L. Guler, R.G. Steen, A.J. Mackey, M.J. Daly, K. Frederick, W.F. Dietrich, and K.M. Murphy. 1996. Genetic mapping of a murine locus controlling development of thelper $1 \mathrm{t}$ helper 2 type responses. Proc. Natl. Acad. Sci. USA. 93:12467-12472.

42. Segal, B.M., and E.M. Shevach. 1996. IL-12 unmasks latent autoimmune disease in resistant mice. J. Exp. Med. 184:771-775.

43. Sundvall, M., J. Jirholt, H.T. Yang, L. Jansson, A. Engstrom, U. Pettersson, and R. Holmdahl. 1995. Identification of murine loci associated with susceptibility to chronic experimental autoimmune encephalomyelitis. Nature Genet. 10:313-317. 